\title{
LVI. Electrical theories of matter and their astronomical consequences with special reference to the principle of relativity
}

\section{A.S. Eddington M.A. F.R.S.}

To cite this article: A.S. Eddington M.A. F.R.S. (1918) LVI. Electrical theories of matter and their astronomical consequences with special reference to the principle of relativity , Philosophical Magazine Series 6, 35:210, 481-487, DOI: 10.1080/14786440608635790

To link to this article: http://dx.doi.org/10.1080/14786440608635790

曲 Published online: 08 Apr 2009.

Submit your article to this journal $[\pi$

Џ Article views: 3

View related articles 
three respects; this involves trigonometric computation. Most important of all is the question how best to utilise the above methods in order to make a much better attempt for the next trigonometric computation, instead of striking out blindly.

Two other similarly computed objectives are :-

(1) For $u_{1}=-0.5$ (object equal to image): $c_{1}=0.8351$, $c_{2}=-4.0075, c_{3}=-3.9539, c_{4}=-1.5224$.

(2) For $u_{1}=-1 \cdot 0$ (object, at focus): $c_{1}=0.0414, c_{2}=$ $-4 \cdot 8012, c_{3}=-4 \cdot 7498, c_{4}=-2 \cdot 3183:$ this may be regarded as corrected for infinity, but with flint leading.

LVI. Electrical Theories of Matter and their Astronomical Consequences with special reference to the Principle of Relativity. By A S. EDdingron, M.A., F.R.S., Plumian Professor of Astronomy in the University of Cambridge**

$\mathbf{M}^{\mathrm{H}}$

R. WALKER'S paper in the Philosophical Magazine for April has given a new turn to the discussion arising out of the motions of the perihelia of the planets, and perhaps some remarks on the points raised may be useful. I think that he greatly overestimates the differences of opinion between us; our views seem to coincide on what he calls "the main point at issue," and it is difficult to believe that relativists in general hold any other view.

Walker describes my method of dealing with the problem as depending on an unsatisfactory assumption, and concludes therefore that my treatment is invalid; but $I$ am afraid he gives a wrong impression by not mentioning that my argument tended to disprove the assumption in question. I did not advocate the assumption leading to the equations of motion used in my paper ; on the contrary I showed that the results disagreed with observation. The suggestion had been made that the famous discordance of Mercury conld be accounted for by the variation of mass with velocity, according to the well-known hypothesis $m=m_{0}\left(1-w^{2} / c^{2}\right)^{-\frac{1}{2}}$, if account be taken of its interaction with the sun's motion through the ather. This hypothesis was examined, and the conclusion was unfavourable, the detailed results being irreconcilable with astronomical observation. That is to say, I attempted to disprove the hypothesis $m=m_{0}\left(1-v v^{2} / c^{2}\right)^{-\frac{1}{2}}$ which Walker rejects more summarily. Sir Oliver Lodge also concludes that "If therefore the theory fails to give all the known perturbations correctly, something must be wrong;

* Communicated by Sir Oliver Lodge. 
and by finding out what is wrong, we may perhaps discover something instructive"*. We may therefore start from this point of general agreement.

The foregoing law of inertia corresponds to the Lorentz electron in steady motion, but the method applies equally to any other law of variation of inertia with velocity; the only difference is a numerical factor which is of no consequence to the argument. A fundamental revision of the theory is therefore necessary. Walker has stepued in with the suggestion that a trial should be made with the more general (and, I consider, more plausible) assumption that the inertia involves the acceleration as well as the velocity. I cannot predict whether his mode of developing this view will lead to an accordance with observation; but I certainly do not undertake to prove a general negative.

Walker's thesis that the Lagrangian function depends on the acceleration as well as on the velocity cannot be a point of cleavage between relativity dynamics and non-relativity dynamics-if I have rigbtly grasped the meaning of the statement. In Newtonian particie dynamics, and also in the quasi-stationary treatment of these problems, the Lagrangian function is supposed to consist of two parts, (1) the kinetic energy, involving the velocity only, and (2) the forcefunction involving position in the field of force. The contention is that this separation is inadmissible, and that there is a cross-term involving both the velocity and the force (or acceleration). Walker's standpoint tends to associate this cross-term with the kinetic energy, so that the kinetic energy differs from the value calculated without regard to the acceleration. Sir Oliver Lodge $\dagger$ and the writer find it more natural to group the term with the force-function, and say that the force of gravitation involves a term depending on the velocity. The distinction appears to be purely rerbal.

It is essential to an out-and-out relativity theory that this cross-term should exist, and it is surprising to find relativists represented as opposed to it.

Nor can the quasi-stationary assumption be regarded as a fundamental point of difference. It would, I think, be absurd either to affirm or deny the quasi-stationary principle irrespective of the particular application proposed. The question is whether it is a legitimate approximation in $a$ definite problem. I sympathize with Walker in demanding a justification of this approximation in the cases where it has been used-whether by relativists or others. The problem of

* Phil. Mag. February 1918, p. 143.

$\dagger$ Loc. cit. pp. 155-156. 
the motion of the planets seems to afford a good illustration of its fallibility. But it seems rather unfair to blame relativists for a method which was introduced by Abraham in a non-relativity theory. I am not sufficiently versed in the history of the subject to know how extensively relativists have followed his example; but I should have regarded it as one of the sins of our youth-due to the influence of evil associates-and long since repented.

I understand that the true relativity theory of Kaufmann's experiment (which seems to be the point in dispute) runs something like this:-Consider an electron momentarily at rest, but continually accelerated, in an electric field of force $\mathrm{E}$ and a magnetic field $\mathrm{H}$; then the acceleration $\alpha$ is given by the equation

$$
\mathrm{E} e=m \alpha,
$$

where $e / m$ is a certain universal constant for the negative electron; we need not inquire into its nature. This equation expresses the ordinary definition of $E$. Now choose new axes of coordinates with respect to which the electron has an instantaneous velocity $w$. Referred to these axes the electric and magnetic forces take known values $\mathrm{E}^{\prime}, \mathrm{H}^{\prime}$, and the new value of the acceleration $\alpha^{\prime}$ is obtained by making Lorentz's transformation of the coordinates and the time. Accordingly the relativity-theory predicts that an electron moving with velocity $w$ in an electromagnetic field $\mathrm{E}^{\prime}, \mathbf{H}^{\prime}$ will experience this acceleration $\alpha^{\prime}$. Keeping $\mathrm{E}^{\prime}$ and $\mathrm{H}^{\prime}$ constant, we find how $a^{\prime}$ depends on $w$. Kaufmann's experiment-or rather the recent repetitions of it-confirm the predicted relation with considerable accuracy. Not only does this give the prestige of successful prediction to the relativity theory, but it confirms that part of the hypothesis most in doubt. It is generally admitted that the Lorentz transformation holds for the differential equations of the field; the question is, Does it hold for the boundary conditions (whatever they may be) at the surface of an electron? The Kaufmann experiment, dealing with a single isolated electron, answers this in the affirmative. If then the differential equations and the boundary conditions satisfy the transformation, nothing more is needed to establish its validity*. It should be noted that the experiment does not

* Experiments are, however, still needed to test whether the Lorentz transformation covers the phenomena of quanta, which appear to involve something ontside the ordinary electromagnetic theory. The exception is of special importance because it includes the vibration of an atom, which is the simplest form of a natural clock that could be used for measuring the time in the two systems. 
tell us what are the boundary conditions at the electron, but only how they are transformed by uniform motion.

There is no reference to the quasi-stationary principle in this theory. It is true also that there is no reference to the mass, energy or momentum of the electron; the motion is troated geometrically. It is, I think, inappropriate to speak of the energy or momentum of an electron in accelerated motion : these quantities are being radiated, and it is impossible to define the precise moment at which an element of energy or momentum ceases to be attached to the electron and passes into the general field. For uniform motion, however, the values can be clearly defined. We do not determine them directly from Kaufmann's experiment; bat we arrive at them indirectly because the relativity transformation is verified. These expressions for the momentum and energy of a uniformly moving electron are of limited utility: as Walker rightly points out, it is not permissible to differentiate them.

I gather from Walker's remarks on p. 329 that he has doubts whether the Fitzgerald-Lorentz contraction should theoretically take place under circumstances such as those of the Michelson-Morley experiment; that is to say, the correlation found by Lorentz and Larmor is a possible one, but it need not necessarily be the correlation occurring in Nature. But a proof based on statistical mechanics has been put forward, which seems to be sound *. The arrangement of the particles constituting a solid is one of an infinite number of possible states, and the form taken up by the solid is that which is statistically most probable; since the possible states of the stationary and moving solid are correlated one to one, the most probable states (and therefore the actual states) satisfy the same correlation. In other words entropy is invariant for the Lorentz transformation.

The relativity principle has the great advantage that it leads directly to the law $m=m_{0}\left(1-w^{2} / c^{2}\right)^{-\frac{1}{2}}$ for uniform motion of matter in bulk, and it is unnecessary to consider the behaviour of an electron, or indeed to adopt an electrical theory of matter. I see no way of deducing from the various electrons treated by Walker the corresponding laws of mass for matter in bulk, so that the discussion of these does not seem to advance the astronomical problem except by suggesting possible analogies.

* E. Cunningbam, 'The Principle of Relativity,' p. 206. Itsewhere this book warns the reader against assuming that the correlation holds for a non-uniform translation; and indeed those who accept Einstein's latest theory assert definitely that it does not hold. 
Turning now to Walker's astronomical calculations, it may be pointed out that his detailed calculations deal with a solar system at rest in the rther. The discussion therefore does not relate to Sir Oliver Lodge's suggestion as to the effects of a solar motion, nor does it throw light on the point subsequently brought out- hat the motion of the solar system (if any) has no observable effect on the motions of Venus and the Earth. At the end of his investigation Walker hints that a satisfactory theory similar to Lodge's might be constructed by using the more general type of Lagrangian function. He has, however, already three unknown constants, $k_{1}, k_{2}, k_{3}$; the components of the unknown solar motion will give him three more; with six constants at disposal, he can scarcely fail to secure a forced agreement of the peribelia and eccentricities of the four inner planets, and it is difficult to find any observational test for such a theory.

On p. 337 it is stated that the observed motion of perihelion of Mercury is satistied by supposing that the attraction depends on the velocity (i. e. relative relocity) according to the law

$$
\mu=\mu_{0}\left(1+{ }_{2}^{5} w^{2} / c^{2}\right),
$$

and that Einstein implicitly introduces this comparatively large dependence on speed. Einstein's law may be transformed in a great many ways; but 1 do not think any possible interpretation of it reduces to this. If it is desired to put the new wine into old bottles, I think we must say that the theory involves different effects of the radial and transverse components of velocity in modifying gravitation, $\mathrm{or}^{*}$ to quote Walker's earlier remark " the modified Lagrangian function depends on the acceleration as well as on the speed of the system and involves also the relative direction of these"*. The point is perhaps not of great importance; because in any case a theory which deduces the exact motion of Mercury from a general principle stands on a different footing from theories which merely use the motion of Mercury to obtain an empiricul determination of their arbitrary constants.

As a closely connected subject, the question of the alleged discordance of the node of Venus deserves some remarks. Dr. Jeffreys (Nature, April 11, p. 103) has commented on

* Walker's method of taking this into account is to give $k_{1}$ and $k_{2}$ appropriate values, but in calculating the number 5 he has used the quasi-stationary values, presumably as a concession to relativists. I am af raid I must reject the concession, and insist on agreeing with his true opinion on this point. 
the scant attention paid to this as compared with the perihelion of Mercury. It may be well to explain why the former discordance has been considered unimportant. The residual of the node of Venus is $4 \frac{1}{2}$ times its probable error *, and the theoretical chances against such an error are about 400 to 1 . But it must not be forgotten that this element has been deliberately selected out of 16 elements as showing the greatest discordance. To apply the test of probable error we must select fairly and not pick out the worst cases. Let $\alpha$ be the probability of an error less than $x$, then the probability that all sixteen residuals are less than $x$ is $\alpha^{16}$. For a limit of $4 \frac{1}{2}$ times the probable error this gives a probability $(\cdot 9976)^{16}=\cdot 962$, so that the chance of the largest residual being as much as $4 \frac{1}{2}$ times the probable error is $\cdot 038$, or about 1 in 26-an adverse probability, but not very emphatic. To put the matter another way, we find (by solving $a^{16}=\frac{1}{2}$ ) that the largest discordance of the 16 elements should just exceed 3 times the probable error. We may therefore ask, What is the probability that Newcomb anderestimated his errors in the ratio 2 owing to unsuspected sources of error? The evidence for a genuine discordance seems very flimsy. To the astronomer, no doubt, it is an indication well worth looking into; but it would be extremely rash to build a theory on so slight a foundation.

The present state of the problem of the elements of the four inner planets appears to be as follows:-The theory given in Sir Oliver Lodge's and my own papers leads to secular perturbations of the Earth and Venus, which ought to be perceptible to observation if the sun's motion is greater than abont $10 \mathrm{~km}$. per sec. Since these are not observed, we conclude either that the sun's motion happens to be very small, or that there must be compensating terms in the more complete theory. Following ont the second alternative, there are again two possibilities. Either the compensation is an accident due to the particular elements of the orbits and their relation to the direction of the sun's motion, or it is a general compensation. A theory can no doubt be constructed which gives an accidental cancelling for Venus and the Tarth, provided it contains a sufficient number of disposable constants not otherwise determined; but such a theory cannot carry much conviction. If we suppose that the compensation is general, then we are adopting effectively a relativity theory of gravitation-that uniform motion of a gravitating system produces no observable effects. This involves a dependence

* The discordance of the perihelion of Mercury from ti: Newtonian theory is 30 times its probable error. 
of gravitation on the velocity of the planets; or, if preferred, the same thing may be expressed in Walker's phraseology.

In this last case the motion of the sun can have no influence on the perihelion of Mercary, and the observed excess must be ascribed either to outside causes, such as the mass or resistance* of the Zodiacal Light, or to the laws of relative motion. In my paper only $\frac{1}{6}$ of the observed excess $\dagger$ is given by the relative motion; and the difficulty is to bring it up to the required amount without extravagant or ad hoe assumptions. Walker's investigation appears ratber to emphasize this difficulty. Nevertheless Einstein's generalized relativity theory gives the precise value required without any arbitrary constants. It may perhaps be said that the factor 6 (or 3 ) must be implicitly contained in the assumptions of his theory. I suppose that the results of any theory are implicitly contained in its postulates; so I cannot deny that the factor 6 is concealed in Einstein's Principle of Equivalence- " that it is impossible by any experiment to discriminate between a gravitational field and a field of force (such as the centrifugal force) arising from a transformation of the coordinates of reference." But at least. it is cleverly camouflaged! Many pages of analysis are required to obtain the result for Mercury, and I do not think any simple interpretation of the occurrence of the factor can be given at present.

LVII. On the Relation of the Audibility Factor of a Shunted Telephone to the Anterna Current as used in the reception of Wireless Signals.

To the Editors of the Philosophical Magazine.

\section{Gentlemen, 一}

TN your January number you published a paper with 1 the above title in which $I$ criticised a previous communication from $\mathrm{Mr}$. van der $\mathrm{Pol}$; my paper was followed by an explanatory note in which Mr. van der Pol attempted to justify his methods and conclusions. He suggests that I had rather lost sight of the motives of his paper and of certain experimental difficulties; I wish to show, however, that, through depending too much on Prof. Love's account of Austin's work and apparently

* Sir Oliver Lodge, ' Nature,' March 21, p. 44.

$\dagger$ Or $\frac{1}{3}$, if the added inertia is subject to gravitation. 